of confinement, with a correction to allow for the Coulomb interaction ${ }^{5}$ between the electrons and holes. The luminescence of colloidal semiconductors is also strongly affected by particle size ${ }^{6}$. Typically, a broad emission is observed which blueshifts with decreasing radius of the aggregate.

The synthesis of colloidal semiconductors is often carried out in solution by chemical precipitation. Because larger aggregates are thermodynamically more stable than smaller ones, it is necessary to arrest the growth process at a stage where the particles are still in the quantum-size domain. This is usually done by adding a protective agent, such as hexametaphosphate, to the solution. However, this method gives a rather broad distribution of particle size, and tedious separation steps are required in order to obtain clusters with fairly uniform morphology.

Herron et al. ${ }^{\prime}$ use an ion exchange procedure to incorporate $\mathrm{Cd}^{2+}$ into the zeolite subsequently exposed to hydrogen sulphide gas to form the CdS microcrystals. An important finding from their detailed $\mathrm{X}$-ray analysis concerns the location of the CdS clusters. At low concentrations, the $\mathrm{Cd}^{2+}$ ions are preferentially adsorbed at the I' site of the zeolite Y (Fig. 2a), so that the clusters are located in the smaller sodalite cages and not, as one might expect, the supercages. The overall structure of the sodalite-cage-entrapped species is a distorted cube containing the tetramer $(\mathrm{CdS}, \mathrm{O})_{4}$ (Fig. $\left.2 b\right)$. The unusual stability of these extremely small clusters is due to the coordination of the $\mathrm{Cd}^{2+}$ ions with oxygen of the zeolite framework. These isolated $\mathrm{CdS}$ clusters are distinguished by their unique optical properties which deviate greatly from those of bulk CdS. Thus, the absorption threshold in their spectrum is around $350 \mathrm{~nm}$, compared to $540 \mathrm{~nm}$ for bulk CdS. The absence of any luminescence emission, even at temperatures down to $4 \mathrm{~K}$, is also conspicuous as large $\mathrm{CdS}$ particles show intense emission under similar conditions.

These optical properties undergo drastic changes at higher $\mathrm{CdS}$ loadings for which adjacent sodalite cages become occupied by the clusters. The absorption threshold shifts to $420 \mathrm{~nm}$ and an excitonlike feature (bound electron-hole 'atom') evolves at $350 \mathrm{~nm}$. Strong emission appears owing to charge-carrier recombination on vacancies and lattice imperfections. These phenomena are ascribed to the formation of CdS 'superclusters' by a percolative process involving aggregation of the tetramers through the double six-rings linking the sodalite moieties. The novel feature of the superclusters is that every $\mathrm{CdS}$ molecule in the aggregate is on the surface and that strong coupling with the host lattice occurs through the interaction between $\mathrm{Cd}$ and the framework oxygen. This explains the unique optical

\title{
Knotty problems in cosmology
}

Numerical studies of the gravitational interaction of collections of point masses have been an important tool in 'experimental' cosmology for some 20 years - such interactions underlie the structure of the Universe at all scales. But many computational difficulties remain. To simulate a respectable fraction of the Universe with a limited number of particles, each particle must represent the mass of many galaxies, but now that observational cosmologists are beginning to map complete galaxy distributions throughout large regions of the Universe, the simulators can keep up their side of the work only by following their models at the level of individual galaxies. This means using enormous numbers of particles, which demands huge allotments of computing time.

A common compromise has been to put in large particle numbers, but then to approximate the behaviour of dense regions, as they form, with some kind of fluid mechanical treatment. But S. F. Shandarin (Institute of Physical Problems, Moscow) and A. Melott, working together at the University of Kansas, have returned to the simple but accurate device of calculating individual trajectories for each of 512 $\times \mathbf{5 1 2}$ particles on a two-dimensional grid of comparable spatial resolution. Their technique demands too much computing power to achieve useful results in three dimensions, but reveals details which, were fewer particles used, would be invisible.

The scale of the simulations is arbitrary,

properties of these composites. It is noteworthy that $\mathrm{CdS}$ clusters in zeolite are distinguished from conventional colloids also by their selective intervention in photocatalytic processes ${ }^{7}$

Dameron et al. describe in this issue $\mathrm{e}^{2}$ the biosynthesis of quantum-sized CdS crystallites in the yeast cells Candida glabrata and Schizo saccharomyces pombe. When exposed to cadmium ions, these cells respond with the synthesis of $\gamma$-glutamyl peptides, $(\gamma$-Glu-Cys) - Gly and an enhancement of sulphide production. As a result, small crystallites of $\mathrm{CdS}$ are produced inside the cells. Surprisingly, the colloids crystallize in the rock-salt structure instead of the hexagonal configuration which is the thermodynamically stable form of CdS. The role of the peptide is that of a protective agent: the cysteinyl thiolates in the peptides are surface ligands to the core of around $85 \mathrm{CdS}$ units. In this way, the organism controls particle but features apparent in the figure resemble structures observed at the scale of superclusters of galaxies. The figure, a detail from one simulation, is colour coded (from red to violet) for the logarithm of the density. The darker regions could correspond to filaments of galaxies

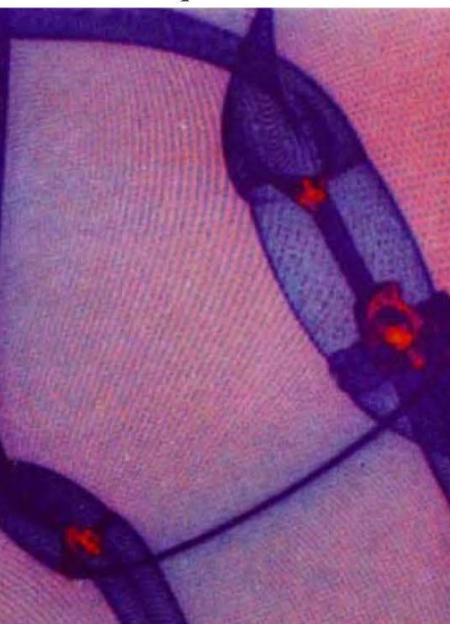

and the intervening patches, 'voids'.

The large structures generally agree with those predicted in catastrophe theory and Zel'dovich's 'pancake' model, but the small-scale structure (such as the voids inside the filaments) is new. Of immediate interest is the suggestion that the small and large structures can be aligned over widely differing length scales. No detailed survey has yet found a scale beyond which the distribution settles to some uniform average. On all dimensions, something seems to be going on, and direct simulations such as these are needed to investigate the controlling influences. David Lindley

nucleation and growth yielding uniformly sized CdS particles of approximately 20 A diameter. Not unexpectedly, the optical absorption of these aggregates reveals pronounced quantum-size effects as in the case of the zeolite contained CdS clusters. The findings of Dameron et al. provide a first example for the biosynthesis of quantum-sized semiconductor crystallites constituting a unique metabolic route for the detoxification of $\mathrm{Cd}^{2+}$-infected living cells.

Michael Grätzel is in the Institut de Chemie Physique, Ecole Polytechnique Federale de Lausanne, CH-1015 Lausanne, Switzerland.

1. Herron, N. et al. J. Am. chem. Soc. 111, 530-540 (1989).

2. Dameron, C.T. et al. Nature 338, 596-597 (1989).

3. Grätzel, M. Heterogeneous Photochemical Electron Transfer (CRC, Boca Raton, Florida, 1989).

4. Berry, C.R. Phys. Rev. 161, 848-855 (1967)

5. Brus, L.E. J. Chem. Phys. 80, 4403-4409 (1984)

6. Papavassiliou, G.C. J. Sol. St. Chem. 40, 330-339 (1981).

Pettit, T.I. thesis, Univ. Texas, Austin (1986) 